\title{
Exploring the Benefit of an Integrated Ophthalmology Internship (PGY-1)-Perceived Preparedness and the Recommended Duration of Training
}

\author{
Andrew Hou, MD ${ }^{1} \quad$ Nikhil Goyal, MD ${ }^{2}$ Deborah Darnley-Fisch, MD ${ }^{1}$ Paul Edwards, MD ${ }^{1}$ \\ David Goldman, MD, MBA ${ }^{1}$ \\ ${ }^{1}$ Department of Ophthalmology, Henry Ford Hospital, Detroit, Michigan
${ }^{2}$ Department of Emergency Medicine and Internal Medicine,
Henry Ford Hospital, Detroit, Michigan \\ Address for correspondence Andrew Hou, MD, Department of \\ Ophthalmology, Henry Ford Hospital, 2799 West Grand Boulevard, \\ Detroit, MI 48202 (e-mail: ahou1@hfhs.org).
}

Acad Oph halmol 2020;12:e57-e60.

J Acad Ophthalmol 2020;12:e57-e60.

\begin{abstract}
Keywords

- residency

- accreditation

- internship

- ophthalmology

- education

- curriculum

- training

- development

Objective The effective utilization of intern training for ophthalmology residency is undermined by its variability across postgraduate programs. As residency programs shift toward integrating the first postgraduate year (PGY-1) into the ophthalmologytraining curriculum, there are no definitive guidelines to build upon.

Methods This is a retrospective cross-sectional study of new second postgraduate year (PGY-2) ophthalmology residents. Residents were surveyed in their first 2 months of ophthalmology training. Residents were asked to self-assess preparedness for ophthalmology based on their PGY-1 curriculum. A survey was composed and modified from the Accreditation Council for Graduate Medical Education's assessment of resident training. The Wilcoxon's rank-sum test was used to evaluate survey differences between the transitional year (TY) versus preliminary internal medicine (IM) year and compare survey responses between residents who were below versus above optimal cut points for weeks of ophthalmic training.

Results There were 72 PGY-2 residents who responded to blinded surveys collected from July to August of 2017 and 2018. Thirty-nine (54\%) residents graduated from a TY, 28 (39\%) from preliminary IM, 3 (4\%) from preliminary surgery, and 2 (3\%) from a categorical ophthalmology year. Both categorical ophthalmology and surgery year were excluded from training year comparison due to low sample size. Using weeks of ophthalmology training as the control variable, there were significant increases in preparedness for addressing the ophthalmic complaint $(p=0.003)$ with at least 8 weeks of clinical ophthalmology, ability to perform ophthalmic exam $(p=0.018)$ with at least 12 weeks, ophthalmology medical knowledge $(p=0.005)$ with at least 10 weeks, and proficiency with hospital electronic health record $(p=0.003)$ with at least 12 weeks.

Conclusion While our study did not find significant differences in perceived preparedness for ophthalmology residency based on the type of PGY-1 programs completed, there were statistically significant associations for specific ophthalmology tasks. These findings suggested a potential merit of integrating 12 weeks of clinical ophthalmology training as a benchmark for resident preparedness.
\end{abstract}

received

March 27, 2019

accepted

March 1, 2020
DOI https://doi.org/

10.1055/s-0040-1709671. ISSN 2475-4757.
Copyright $\odot 2020$ by Thieme Medical Publishers, Inc., 333 Seventh Avenue, New York, NY 10001, USA. Tel: +1(212) 760-0888.
License terms

()(1) $\Theta \circledast$ 
The prevailing paradigm in ophthalmology training is the 1-year internship followed by 3 years of residency. Currently, there is high heterogeneity in the models and approaches to clinical and surgical training across the programs in the United States. As more programs recognize that older apprenticeship models require higher overall cost and time to develop technical skills, there will be a need to enhance the capacity of residency training time. ${ }^{1}$

Currently, the variability in the first year of intern training limits the timely utilization of postgraduate training for ophthalmology residency. Ophthalmology-bound interns can complete their first postgraduate year (PGY-1) in emergency medicine, family medicine, internal medicine (IM), neurology, obstetrics and gynecology, pediatrics, surgery, or a transitional year (TY). ${ }^{1}$ The need for optimizing training programs becomes paramount because of the continuous evolution of knowledge and technology. As residency programs shift toward the consideration of integrating the PGY1 with the ophthalmology residency, there is no definitive guide as to how to create the PGY-1 curriculum. An important question to consider is whether or not a minimum recommended ophthalmology exposure time during PGY-1 results in an increased sense of readiness for the ophthalmology second postgraduate year (PGY-2) demands and responsibilities.

\section{Methods}

This study was a multicenter retrospective observational crosssectional survey of PGY-2 ophthalmology residents enrolled in Accreditation Council for Graduate Medical Education (ACGME)-accredited training programs in the United States. The survey was developed at the Henry Ford Health System and sent to residents across the country through the residency program director electronic mailing list. The initial question prior to beginning the survey regarded informed consent. It acknowledged that the survey would remain completely anonymous, provide confidentiality, and prevent programs from identifying survey responders. The study method and design were reviewed and approved by the Institutional Review Board for Human Studies at Henry Ford Hospital.

\section{Survey Development}

The survey was based on a modified assessment of resident training from the ACGME. ${ }^{2}$ The survey participants self-identified which type of PGY-1 program they completed. TY is defined by the ACGME as an intern program for a career specialty that requires, as a prerequisite, 1 year of clinical education. Preliminary year is defined as a position offering only 1 to 2 years of training generally prior to entry into advanced specialty programs. Preliminary years are most commonly offered in IM or surgery. Categorical year offers full residency training required for board certification in that specialty.

The survey questions asked for subjective preparedness in clinical skills such as addressing the complaint, performing the ophthalmic exam, selecting and interpreting diagnostic tests, identifying diagnoses, and choosing therapies. The survey also assessed professional skills such as communication in regard to consultation, handoffs, teamwork, and didactics. This survey did not evaluate surgical skill preparedness.

Supplementary Appendix 1 shows the questions as well as the available answer choices.

\section{Survey Distribution}

The survey was distributed electronically using an online survey tool (SurveyMonkey, San Mateo, CA). An e-mail was sent to program coordinators who are members of the Association of University Professors of Ophthalmology with a request to forward it to their residents. Residents were surveyed in their first 2 months of ophthalmology training. The responses were collected from July through August of 2017 and 2018. The surveys were tagged with a responder's unique IP address to ensure that there were no duplicate surveys, but no unique hyperlinks were attached to ophthalmology programs to maintain anonymity.

\section{Data and Statistical Analysis}

Comparisons were made between data collected from intern trainees across the two main clinical training platforms of TY and preliminary IM. Preliminary surgery and categorical year data were excluded, as the sample size was too small to allow for accurate analysis. The Wilcoxon's rank-sum test was used to compare the survey responses of the two groups. In addition, the Wilcoxon's rank-sum test was used to compare survey responses between all residents who were below versus above optimal cut points of weeks of PGY-1 clinical ophthalmology training. A $p$-value of less than 0.05 was considered a significant relationship.

\section{Results}

Of the 931 survey invitations sent, 72 PGY-2 residents $(7.73 \%$ response rate) returned anonymous surveys during July toAugust of 2017 and 2018. Thirty-nine (54\%) graduated from TY, 28 (39\%) from preliminary IM, 3 (4\%) from preliminary surgery, and 2 (3\%) from categorical ophthalmology year programs.

Categorical ophthalmology and surgery year were excluded from the training year comparisons due to their low sample size. We did not find any statistically significant differences in preparedness variables when comparing training under the TY or IM training year (- Table 1). However, using weeks of PGY-1 ophthalmology training as the control variable, within the full set of residents there was a significant increase in self-perceived preparedness for specific skills with increased exposure to ophthalmology during the internship year. These included addressing the ophthalmic complaint ( $[p=0.003]$ after at least 8 weeks of clinical ophthalmology), ability to perform ophthalmic exam ( $[p=0.018]$ after at least 12 weeks), ophthalmology medical knowledge ( $[p=0.005]$ after at least 10 weeks), and proficiency with hospital electronic health record ( $[p=0.0 .003]$ after at least 12 weeks). - Table 1 shows the TY versus IM results for subjective level of preparedness. - Table 2 shows the minimum number of weeks of clinical ophthalmology at 
Table 1 Survey comparisons of the transitional versus preliminary medicine programs

\begin{tabular}{|l|l|l|l|}
\hline \multirow{2}{*}{ Survey item } & \multicolumn{2}{l}{ Setting } & \multirow{2}{*}{ Wilcoxon's rank-sum test, $p$-value } \\
\cline { 2 - 3 } & Transitional & Preliminary medicine & \\
\hline Medical school exposure & $2.31 \pm 1.10$ & $2.32 \pm 1.49$ & 0.870 \\
\hline Weeks of PGY-1 clinical ophthalmology training & $5.46 \pm 4.67$ & $3.18 \pm 3.15$ & $0.027^{\text {a }}$ \\
\hline Addressing the ophthalmic complaint & $0.90 \pm 0.85$ & $0.75 \pm 0.75$ & 0.514 \\
\hline Performing the ophthalmic exam & $0.85 \pm 0.84$ & $0.86 \pm 0.93$ & 0.984 \\
\hline Handling on-call consultation & $0.18 \pm 0.39$ & $0.14 \pm 0.45$ & 0.465 \\
\hline Choosing diagnostic procedures & $0.64 \pm 0.71$ & $0.64 \pm 0.73$ & 0.983 \\
\hline Obtaining correct diagnoses & $1.15 \pm 0.78$ & $0.89 \pm 0.74$ & 0.187 \\
\hline Managing nonsurgical treatments & $0.46 \pm 0.64$ & $0.64 \pm 0.91$ & 0.575 \\
\hline Managing minor nonsurgical procedures & $0.26 \pm 0.44$ & $0.21 \pm 0.50$ & 0.516 \\
\hline Handling consultations & $0.64 \pm 0.63$ & $0.71 \pm 0.71$ & 0.765 \\
\hline Ophthalmic medical knowledge & $0.77 \pm 0.67$ & $0.57 \pm 0.50$ & 0.267 \\
\hline Proficiency with electronic record & $1.87 \pm 1.06$ & $1.75 \pm 1.11$ & 0.667 \\
\hline Communicating handoffs and errors & $0.85 \pm 0.63$ & $0.86 \pm 0.76$ & 0.983 \\
\hline Effectiveness/comfort with teamwork & $1.18 \pm 0.82$ & $0.96 \pm 0.84$ & 0.298 \\
\hline Presenting didactic and case-based material & $0.72 \pm 0.65$ & $0.68 \pm 0.72$ & 0.727 \\
\hline
\end{tabular}

Abbreviation: PGY, postgraduate year.

Note: Proficiency can range from 0 to 4 , with $0=$ the worst preparedness and $4=$ the best preparedness. Data are given as mean \pm standard deviation.

aStatistically significant, $p<0.05$.

Table 2 Survey comparisons for optimal PGY-1 ophthalmic training cut points

\begin{tabular}{|l|l|l|l|l|}
\hline Survey item & $\begin{array}{l}\text { Optimal PGY-1 ophthalmic } \\
\text { training cut point }\end{array}$ & \multicolumn{2}{l|}{ Cut point grouping } & Wilcoxon's rank-sum \\
test,p-value
\end{tabular}

Abbreviation: PGY, postgraduate year.

Note: Proficiency can range from 0 to 4 , with $0=$ the worst preparedness and 4 = the best preparedness.Data are given as mean \pm standard deviation.

${ }^{a}$ Statistically significant, $p<0.05$.

which there was a significant preparedness difference noted for all residents.

\section{Discussion}

Our study explored the association in perceived preparedness with respect to the type of PGY-1 training program residents completed. In addition, the study explored the association between the numbers of weeks of clinical ophthalmology training with perceived preparedness when starting the PGY-2. As residency programs across the United States continue to evaluate the PGY-1 model, informed decisions must be made to construct the new paradigm for ophthalmology training. The concept of integrating PGY-1 programs is not a novel idea as other specialties have moved away from preliminary years to minimize transition time and maximize resident learning and proficiency. ${ }^{3,4}$ Our study found that 12 weeks of clinical ophthalmology during PGY-1 was associated with a statistically significant difference in resident perception of preparedness for several important skills. These included addressing the ophthalmic complaint, performing the eye exam, and knowledge base of ophthalmic conditions. Additionally, the study showed that residents perceived that they needed 12 weeks to feel proficient in using the ophthalmology electronic health record system. Eliminating this transition alone can free up significant time for other aspects of clinical learning and improve clinical efficiency. ${ }^{5}$

Interestingly, our study showed that the correlation between the time of ophthalmology exposure during PGY-1 and a perceived improvement to the training experience of residents and preparedness for PGY-2 did not change for cut points beyond 12 weeks. 
Finally, the study inferred that the number of PGY-1 weeks of ophthalmology training did not appear to influence perception of preparedness for several other factors. These included the handling of consultations, handoffs, diagnosis, and management of diseases. It is our hypothesis that this is likely due to PGY-1 residents often assuming the role of "rotators" with the purpose of learning subject matter without being ultimately responsible for the patients' care. However, the integration of residency training may allow for earlier transition to ophthalmology specific on-call duties and clinical responsibilities while facilitating earlier proficiency. ${ }^{6,7}$ Current programs that have already implemented an integrated PGY-1 program can attest anecdotally that these skills are enhanced by the additional ophthalmology time during the PGY-1.

Although our findings suggested the merit of integrating 12 weeks of clinical ophthalmology training as a benchmark for resident perceived preparedness, there were significant limitations to our study. The study was purely subjective in nature and did not objectively measure competency or preparedness. In addition, the study was not powered to ensure adequate comparison between the different PGY-1 models. Finally, we did not sample residents who completed a PGY-1 in pediatrics or obstetrics/gynecology as the low number of residents completing these programs nationwide would have likely precluded analysis.

Another limitation of the study is that the curriculum of ophthalmology months was not qualitatively assessed. There is no universally accepted structure for ophthalmology training months in the PGY-1, and programs may have high variability in the didactics and clinical training provided in a given time frame. ${ }^{8,9}$

\section{Conclusion}

Further comparative studies are needed to gather an adequate sample size to compare an integrated ophthalmology residency versus the current paradigm. As residency programs assert greater control of the PGY-1 training year, it will be vital to standardize the expectations and experience. Though residency programs will ultimately vary in their educational design and structure, the goal is to ensure that
United States' ophthalmologists complete their training with the best possible skillsets for providing outstanding patient care.
Funding
None.

\section{Conflict of Interest}

None declared.

Acknowledgments

None.

\section{References}

1 ACGME. Common Program Requirements. Available at: https:// www.acgme.org/What-We-Do/Accreditation/Common-Program -Requirements. Accessed: March 1, 2019

2 The Ophthalmology Milestone Project. Available at: https://www. acgme.org/Portals/0/PDFs/Milestones/OphthalmologyMilestones.pdf. Accessed: March 1, 2019

3 Oetting TA, Alfonso EC, Arnold A, et al. Integrating the internship into ophthalmology residency programs: association of university professors of ophthalmology American Academy of Ophthalmology White Paper. Ophthalmology 2016;123(09):2037-2041

4 Pfeifer CM. Evolution of the preliminary clinical year and the case for a categorical diagnostic radiology residency. J Am Coll Radiol 2016;13(07):842-848

5 Thomsen ASS, la Cour M, Paltved C, et al. Consensus on procedures to include in a simulation-based curriculum in ophthalmology: a national Delphi study. Acta Ophthalmol 2018;96(05):519-527

6 Roohipoor R, Yaseri M, Teymourpour A, Kloek C, Miller JB, Loewenstein JI. Early performance on an eye surgery simulator predicts subsequent resident surgical performance. J Surg Educ 2017;74(06):1105-1115

7 Daly MK, Gonzalez E, Siracuse-Lee D, Legutko PA. Efficacy of surgical simulator training versus traditional wet-lab training on operating room performance of ophthalmology residents during the capsulorhexis in cataract surgery. J Cataract Refract Surg 2013;39(11):1734-1741

8 Puri S, Srikumaran D, Prescott C, Tian J, Sikder S. Assessment of resident training and preparedness for cataract surgery. J Cataract Refract Surg 2017;43(03):364-368

9 Liu EY, Li B, Hutnik CML. Canadian ophthalmic microsurgery course: an innovative spin on wet lab-based surgical education. Can J Ophthalmol 2016;51(05):315-320 